\title{
EFFICIENT KNOWLEDGE ASSESSMENT BASED ON CONVEX GEOMETRIES
}

\author{
Sylvia Encheva \\ Stord/Haugesund University College \\ Bjornsonsg. 45, 5528 Haugesund, Norway \\ sbe@hsh.no
}

Sharil Tumin

University of Bergen

IT-Dept, P. O. Box 7800, 5020 Bergen, Norway

edpst@it.uib.no

\begin{abstract}
The goal of this paper is to develop a theoretical framework for efficient assessment of leamers' understanding of carefully chosen terms and concepts. The model is based on the theory of knowledge spaces and lattices of convex geometries. The strueture of the latter is used to select only knowledge states that imply understanding of key ideas and minimize the effect of lucky guesses while determining leamer's knowledge.
\end{abstract}

Keywords: Knowledge, lattices

\section{Introduction}

Assessing the initial knowledge of a student and updating this assessment as the student progresses through a course is an important part of an intelligent tutoring system. A framework for representing and measuring students' knowledge is developed in [7]. The key concepts in the theory of knowledge spaces are the knowledge state - a subset of problems that an individual is capable of solving correctly, and the knowledge structure - a distinguished collection of knowledge states [10].

Establishing the knowledge state of a student in a subject may be require a long sequence of questions. Therefore it might not be very effective for providing immediate help to the student while he/she is working on a particular part of the curriculum. In this paper we propose a model for automated assessment 
of learner's understanding of comparatively small units that are considered to be fundamental in a subject. The model is based on knowledge spaces and lattices of convex geometries.

Systems like 'Assessment and LEarning in Knowledge Spaces' (ALEKS) [16] and Relational Adaptive Tutoring Hypertext (RATH) [17] aim at establishing the knowledge state of each student in a certain knowledge domain and then provide further guidance and personalized help.

This model differs from existing systems in the following:

- it is based on multiple choice tests,

- can assess high level thinking,

- rewards partial knowledge,

- does not apply coefficients for guessing correction, and

- knowledge states are arranged in meet-distributive lattices and student's understanding of an atom is found satisfactory if his/her response belongs to a sublattice of a lattice of the convex geometries on the set of related atoms.

Tests are designed to assess critical thinking applying Bloom's Taxonomy [2]. Such tests contain stems asking students to identify the correct outcome of a given circumstance, map the relationship between two items into a different context, respond to what is missing or needs to be changed within a provided scenario, and evaluate the proposed solution based upon criteria provided.

The rest of the paper is organized as follows. Related work is listed in Section 1. Selected theory used for the model development is presented in Section 2. The model description can be found in Section 3. The paper ends with a conclusion placed in Section 4 .

\section{Related Work}

A model for student knowledge diagnosis through adaptive testing is presented in [13]. Permutational multiple choice question tests have been used for assessing high-level thinking [11]. Students' conceptual thinking can be assessed by presenting them with tests where all the correct answers should be chosen and/or answers require integration of several components or approaches [3] and [11].

The use of formula in a spreadsheet to convert the raw assessment marks into marks or grades corrected for guessing or additionally allowing for the maximum expected mark is demonstrated in [14].

An excellent introduction to ordered sets and lattices and to their contemporary applications can be found in [5]. 
Subsets of relevent examination questions and certain skills from a branch of knowledge are listed as examples of knowledge states in [8]. They are followed by an important remark that not all possible subsets of such items turn out to be knowledge states.

ALEKS [16] is based on mathematical cognitive science and involves computer algorithms while constructing specific knowledge structures. Markovian procedures are further employed for analyzing of a particular student's knowledge.

RATH [17] combines mathematical hypertext model and knowledge space theory and is focused on teaching.

\section{Preliminaries}

Let $Q$ be a finite set. A family $\mathcal{K}$ of subsets of $Q$ is a knowledge space on $Q$ [7] if

i) the empty set and the total set $Q$ are members of the family $\mathcal{K}$, and

ii) the family $\mathcal{K}$ is closed under union.

An atom at item $q$ in knowledge space theory is a minimal knowledge state containing $q$. A state is called an atom if it is an atom at $q$ for some item $q$.

A base for a knowledge structure $(Q, \mathcal{K})$ is a minimal family $\mathcal{A}$ of states spanning $\mathcal{K}$ ('minimal' with respect to set inclusion, i.e. if $\mathcal{F} \subseteq \mathcal{A}$ is any family of states spaning $\mathcal{K}$, then $\mathcal{F}=\mathcal{A}$ ).

A closure system on a finite set $M$ is a set $\mathcal{F}$ of subsets of $M$ such that

1) $M \in \mathcal{F}$ and

2) $C, C^{1} \in \mathcal{F} \Rightarrow C \cap C^{1} \in \mathcal{F}$

A knowledge space is a closure system [7]. A closure system on set $M$ is convex geometry if it satisfies the following properties:

- the empty set is closed

- for every closed set $M_{1} \neq M$ there exists $m \notin M_{1}$ such that $M_{1}+m$ is a closed set.

A lattice is a partially ordered set, closed under least upper and greatest lower bounds. The least upper bound of $x$ and $y$ is called the join of $x$ and $y$, and is sometimes written as $x+y$; the greatest lower bound is called the meet and is sometimes written as $x \dot{y}$.

$X$ is a sublattice of $Y$ if $Y$ is a lattice, $X$ is a subset of $Y$ and $X$ is a lattice with the same join and meet operations as $Y$. A lattice $L$ is meet-distributive if for each $y \in L$, if $x \in L$ is the meet of (all the) elements covered by $y$, then the interval $[x ; y]$ is a boolean algebra.

Convex geometries are closure systems which satisfy anti-exchange property, and they are known as dual of antimatroids. The set of closed sets of a 

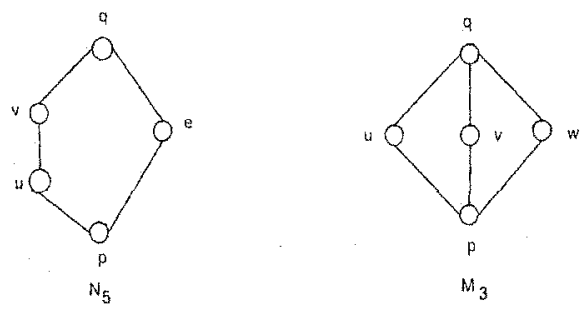

Figure 1. The two lattices $N_{5}$ and $M_{3}$

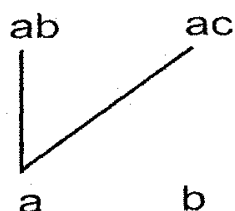

bc

b

C

Figure 2. Rule 1 - one basic question from $\mathcal{B}$ and two related questions from $\mathcal{R}$.

convex geometry, form a lattice when ordered by set inclusion. Such lattices are precisely the meet- distributive lattices.

THEOREM 1 [5] Let L be a lattice.

i) $L$ is non-modular if and only if $L$ has a sublattice isomorphic to $N_{5}$.

ii) $L$ is non-distributive if and only if $L$ has a sublattice isomorphic to $N_{5}$ or $M_{3}$.

\section{A Unit Followed by Six Questions}

After going through a larger unit in a subject, a student is suggested to take a test with six questions. Three of the questions consider understanding of new terms or applying new skills, and are denoted by $\{a, b, c\}=\mathcal{B}$. The other three are denoted by $\{a b, a c, b c\}=\mathcal{R}$ and indicate student's ability to apply both $a$ and $b$ at the same time (denoted $a b$ ), both $a$ and $c$ at the same time (denoted $a c$ ) and both $b$ and $c$ at the same time (denoted $b c$ ). For the sake of presentation simplicity we do not involve more questions. Further more we assume that a student has sufficient knowledge and understanding of a question if he/she gives correct answers to:

- one basic question from the set $\mathcal{B}$, say $c$ and the two related to $c$ questions from the set $\mathcal{R}$, i.e. $a c, b c$ (see Fig. 2), or 


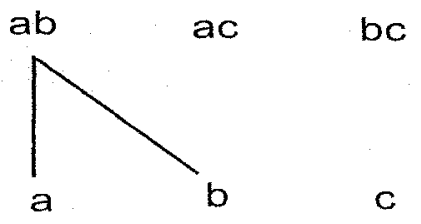

Figure 3. Rule 2 - two basic questions from $\mathcal{B}$ and the related question from $\mathcal{R}$

Table 1. Correct answer combinations leading to a reduced test in a consecutive trial in the case of six questions

\begin{tabular}{|c|c|c|c|c|c|c|}
\hline \multicolumn{6}{|c|}{$\begin{array}{l}\text { Correctly answered questions } \\
\text { in a current test }\end{array}$} & \multirow[t]{2}{*}{$\begin{array}{l}\text { Questions to be excluded in } \\
\text { a consecutive trial }\end{array}$} \\
\hline$a$ & $\mathrm{~b}$ & c & $\overline{a b}$ & $\mathrm{ac}$ & $\overline{b c}$ & \\
\hline \multirow[t]{3}{*}{-} & & & - & - & & $a$ \\
\hline & - & & - & & - & $\mathrm{b}$ \\
\hline & & $\bullet$ & & - & - & $\mathrm{c}$ \\
\hline$\bullet$ & - & & - & & & $a b$ \\
\hline \multirow[t]{2}{*}{-} & & - & & & - & $\mathrm{ac}$ \\
\hline & & $\bullet$ & - & & $\bullet$ & $b c$ \\
\hline - & - & & - & $\cdot$ & & $a, a b$ \\
\hline$\bullet$ & 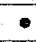 & $\bullet$ & & $\cdot$ & & $\mathrm{b}, \mathrm{ab}$ \\
\hline \multirow[t]{3}{*}{ - } & & - & - & & $\bullet$ & $a, a c$ \\
\hline & $\cdot$ & $\cdot$ & - & & - & $\mathrm{b}, \mathrm{bc}$ \\
\hline & $\bullet$ & $\bullet$ & & - & $\bullet$ & $c, b c$ \\
\hline- & & - & & $\bullet$ & $\bullet$ & $c, a c$ \\
\hline$\bullet$ & $\bullet$ & 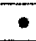 & $\bullet$ & $\bullet$ & & $a, a b, a c$ \\
\hline$\bullet$ & - & - & - & & - & $\mathrm{b}, \mathrm{ab}, \mathrm{bc}$ \\
\hline- & $\bullet$ & $\bullet$ & & $\bullet$ & - & $\mathrm{c}, \mathrm{ac}, \mathrm{bc}$ \\
\hline
\end{tabular}

- two basic question from the set $\mathcal{B}$, say $a, b$ and the related to $a$ and $b$ question from the set $\mathcal{R}$, i.e. $a b$ (see Fig. 3 ).

In other words we want to filter out all answer combinations that do not contain basic questions and the related to them questions, f. ex. $a, b, a c$ in Fig. 4. The lattice in Fig. 4 is a $M_{3}$ lattice from Theorem 1.

Insted of applying one of the numerous ways of penalizing students for guessing we apply the rules illustrated in Fig. 2 and Fig. 3. The outcome is listed in Table 1 and has a graphical representation shown by Fig. 5 .

Case 1:

If a student can answer correctly to less than three questions or to exactly three questions from either $\mathcal{B}$ or $\mathcal{R}$, the system will present $\mathrm{him} /$ her with selected 


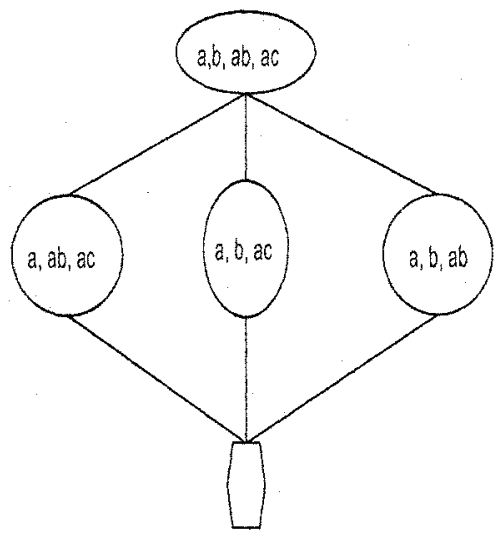

Figure 4. A modular lattice which is not distributive

learning materials (theory and examples). Next time the student takes the same test he/she will be presented with six similar questions but developed by another course builder.

\section{Case 2:}

Suppose a student answers correctly to questions $c, a c, b c$, (see Fig. 6). This answer combination indicates mastering question $c$ and makes no assumptions about other questions. The student will then be advised to work with selected learning materials (theory and examples) concerning questions $a$ and $b$. Next time the student takes the same test he/she will be presented with five questions $a, c, a b, a c, b c$ again developed by another course builder. If the student answers correctly to all of them, the process of questioning is terminated. If the students fails to give correct answers to some of the questions $a, b, a b, a c, b c$ then procedures similar to the following cases will be applied.

\section{Case 3:}

Suppose a student answers correctly to questions $b, c, b c$ (see Fig. 7). This answer combination indicates mastering question $b c$ and makes no assumptions about other questions. The student will then be advised to work with selected learning materials (theory and examples) concerning questions $a, b$ and $c$. Next time the student takes the same test he/she will be presented with five questions $a, b, c, a b, a c$ again developed by another course builder. If the student answers correctly to all of them the process of questioning is terminated. If the students fails to give correct answers to some of the questions $a, b, c, a b, a c$ then procedures similar to the following cases will be applied. 


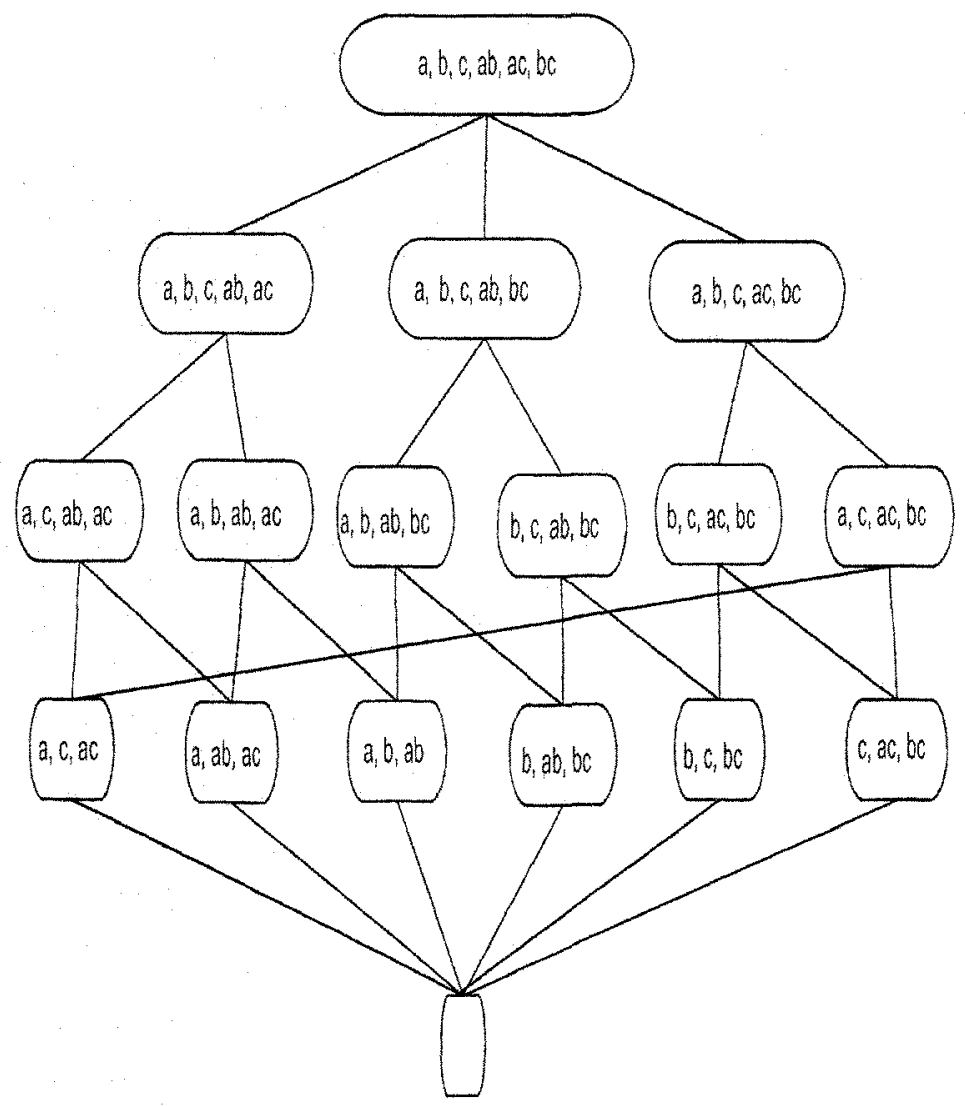

Figure 5. The sublattice of the convex geometries on the basic set of questions $a, b, c$

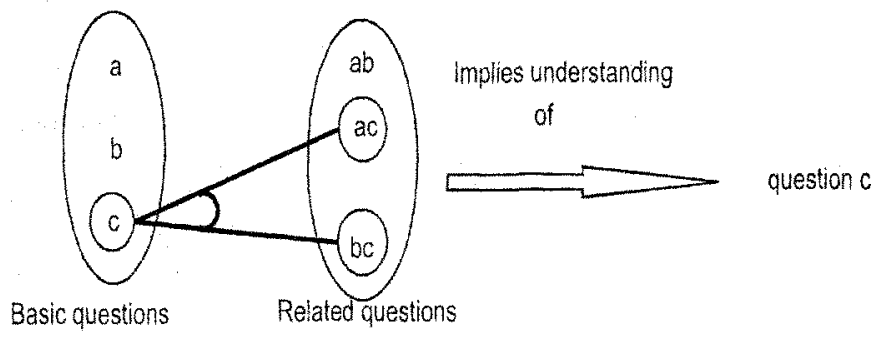

Figure 6. Correct answers to questions $c, a c, b c$ 


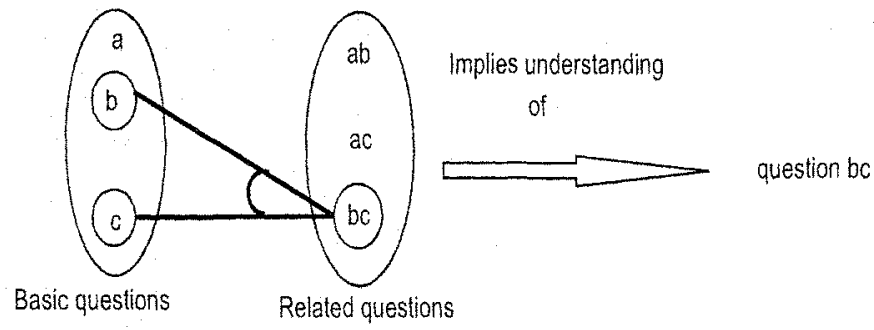

Figure 7. Correct answers to questions $b, c, b c$

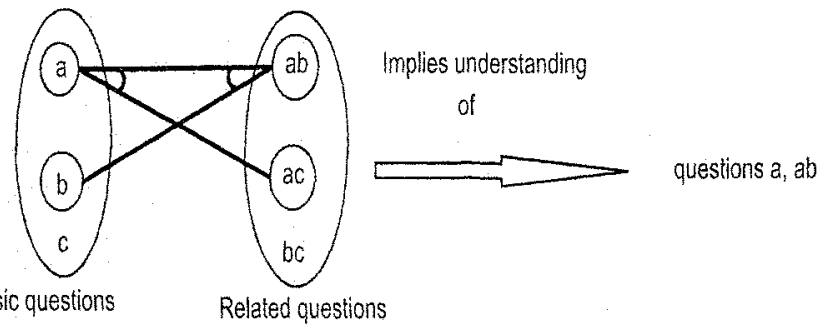

Figure 8. Correct answers to questions $a, b, a b, a c$

\section{Case 4:}

Suppose a student answers correctly to questions $a, b, a b, a c$ (see Fig. 8). This answer combination indicates mastering questions $a$ and $a b$ and makes no assumptions about other questions. The student will then be advised to work with selected learning materials (theory and examples) concerning questions $b$ and $c$. In this case we still repeat question $b$ since by not answering correctly to question $b c$ the student might have a problem applying knowledge from question $b$ to other domains. Next time the student takes the same test he/she will be presented with four questions $b, c, a c, b c$ again developed by another course builder. If the student answers correctly to all of them the process of questioning is terminated. If the students fails to give correct answers to some of the questions $b, c, a c, b c$ then procedures similar to the following case will be applied.

\section{Case 5:}

Suppose a student answers correctly to questions $a, b, c, a b, b c$ (see Fig. 9). This answer combination indicates mastering questions $b, a b$ and $b c$ and makes no assumptions about other domains. The student will then be advised to work with selected learning materials (theory and examples) concerning questions $a$ and $c$. In this case we still repeat questions $a, c$ since by not answering cor- 


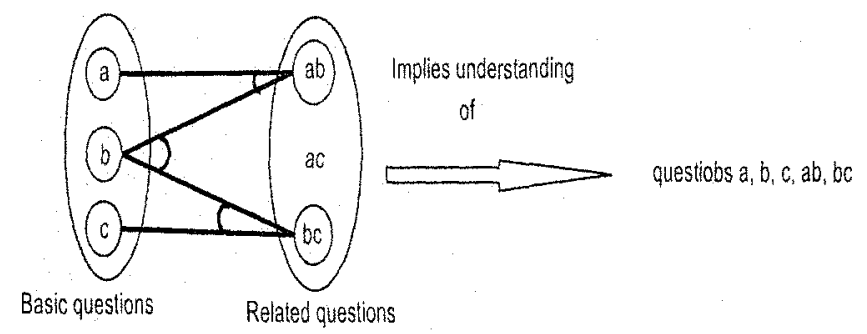

Figure 9. Correct answers to questions $a, b, c, a b, b c$

rectly to question $a c$ the student might have a problem applying knowledge from questions $a, c$ to other arias. Next time the student takes the same test he/she will be presented with three questions $a, c, a c$ again developed by another course builder. If the student answers correctly to all of them the process of questioning is terminated. If the students fails to give correct answers to some of the questions $a, c, a c$ then a similar procedure will be applied.

\section{Conclusion}

A theoretical framework for efficient assessment of learners' understanding of carefully chosen terms and concepts is presented. The model is based on the theory of knowledge spaces and lattices of convex geometries.

\section{References}

[1] D. Albert and J. Lukas (eds.). Knowledge Spaces. Lawrence Erlbaum Associates, 1999.

[2] B. Bloom. The 2 sigma problem: The search for methods of group instruction as effective as one-to- one tutoring. Educational Reseurcher, 13(6):4-16, 1984.

[3] M. Bush. A multiple choice test that rewards partial knowledge. Journal of Further and Higher Education, 25(2):157-163, 2001.

[4] C. Carpineto and G. Romano. Concept Data Analysis: Theory and Applications. Jom Wiley and Sons, L.td., 2004.

[5] B. A. Davey and H. A. Priestley. Introduction to lattices and order. Cambridge University Press, Cambridge, 2005.

[6] J.-P. Doignon and J.-C. Falmagne. Well graded families of relations. Discrete Mathematics, 173(1-3):35-44, 1997.

[7] J.-P. Doignon and J.-C. Falnagne. Knowledge Spaces. Springer-Verlag, 1999.

[8] C.E. Dowling. On the irredundant gencration of knowledge spaces. Journal of Mathematical Psychology, 37, 49-62, 1993.

[9] P.H. Edelman and R.E. Jamison. The theory of convex gcometries. Geom. Dedicata, 19, 247-270, 1985. 
[10] J.-C. Falmagne, M. Koppen, M. Villand, L. Johannesen. Introduction to knowledge spaces: How to build test and search them. Psychological Review: 97, 201-224, 1990.

[11] D.W. Farthing. Permutational multiple choice questions: An objective and efficient alternative to essay-type cxamination questions. Proceedings of the 3rd Anmial Conference on Integrating Technology into Computer Science Education (ITiCSE'98), 1998.

[12] P. Gruber and J. Wills. Handbook of Convex Geometry. North Holland, 1993.

[13] E. Guzman and R. Concjo. A model for student knowledge diagnosis through adaptive testing. Springer-Verlag, LNCS 3220, 12-2,1 Berlin Heidelberg New Jork, 2004.

[14] R. Harper. Correcting computer-based assessments for guessing. Journal of Computer Assisted Learning, 19(1):2-8, 2003.

[15] M. Koppen. Extracting human cxpertise for constructing knowledge spaces: an algorithm. Journal of mathematical psychology, 37, 1-20, 1993.

[16] http:/www.aleks.com

[17] http:/wundt.uni-graz.at/projects/rath 